\title{
ON THE ABSOLUTE HARMONIC SUMMABILITY OF FOURIER SERIES
}

\author{
S. M. SHAH
}

1. Introduction. Let $f(x)$ be a continuous periodic function, period $2 \pi$, and of bounded variation, and let $\omega(\delta)$ denote the modulus of continuity of $f$. It is known that [6, pp. 241-242], if $\sum n^{-1}\left(\omega\left(n^{-1}\right)\right)^{1 / 2}$ is convergent, then the Fourier series of $f$ is absolutely convergent. This result is best possible [2]. In a recent paper [4] Varshney proved that if

$$
|f(x+h)-f(x)| \leqq A \log ^{-1-\epsilon}(1 / h) \quad(\epsilon>0,0 \leqq x \leqq 2 \pi),
$$

then the Fourier series of $f$ is absolutely harmonic summable. In this note we prove the following ${ }^{1}$

THEOREM. If a continuous periodic function $f(x)$, of period $2 \pi$, is of bounded variation over $[0,2 \pi]$ and the series

$$
\sum_{1}^{\infty}(1 / n) \omega(1 / n)
$$

is convergent, then the Fourier series of $f$ is absolutely harmonic summable.

It is easily seen that the condition (1) implies the convergence of the series (2).

2. Lemmas. Lemma 1.

$$
\begin{gathered}
\lim _{x \rightarrow \infty} \omega(1 / x) \log x=0 . \\
\sum_{1}^{\infty} a_{n} \equiv \sum_{1}^{\infty} \frac{1}{n 2^{n / 2}}\left(\int_{1 / \pi}^{2^{n+1} / \pi} \omega^{2}\left(\frac{1}{t}\right) d t\right)^{1 / 2}<\infty .
\end{gathered}
$$

Proof. (i) Denote by $A, A_{1}, \cdots, c, \cdots$ positive constants. We have for $x>x_{0}(\epsilon)$,

$$
\epsilon>\int_{x}^{x^{2}} \frac{1}{t} \omega\left(\frac{1}{t}\right) d t>\omega\left(\frac{1}{x^{2}}\right) \log x,
$$

and (i) follows.

Presented to the Society, January 10,1961 ; received by the editors February 23 , 1961.

${ }^{1}$ See [1] and [3] for similar results, when $f$ is not of bounded variation. 
(ii)

$$
\begin{aligned}
\int_{1 / \pi}^{2^{n+1} / \pi} \omega^{2}\left(\frac{1}{t}\right) d t & <O(1)+\int_{n_{0}}^{2^{n+1} / \pi} \frac{d t}{(\log t)^{2}} \\
& <O(1)+A \frac{2^{n}}{n^{2}} .
\end{aligned}
$$

Hence the convergence of $\sum a_{n}$ follows by comparison with $\sum 1 / n^{2}$. Lemma 2.

$$
\sum_{1}^{\infty} a_{n} \equiv \sum_{1}^{\infty} \omega\left(\pi / 2^{n+1}\right)<\infty
$$

$$
\sum_{1}^{\infty} b_{n} \equiv \sum_{1}^{\infty}(1 / n) \omega^{1 / 2}\left(\pi / 2^{n}\right)<\infty .
$$

Proof. (i) Since the series (2) is convergent, $\sum_{1}^{\infty} \omega\left(1 / 2^{n}\right)<\infty$ and so $\sum a_{n}<\infty$.

(ii) The series $\sum b_{n}$ is convergent if

$$
\sum_{2}^{\infty}(1 / n \log n) \omega^{1 / 2}(1 / n)<\infty .
$$

Now

$$
\sum_{2}^{\infty}(1 / n \log n) \omega^{1 / 2}(1 / n)=\sum_{1}+\sum_{2}
$$

where in $\sum_{1}$ we put all terms for which $(\omega(1 / n))^{1 / 2} \log n<1$, and in $\sum_{2}$ the rest. Then

$$
\sum_{1} \leqq \sum \frac{1}{n(\log n)^{2}}<\infty, \quad \sum_{2} \leqq \sum \frac{1}{n} \omega\left(\frac{1}{n}\right)<\infty .
$$

Lemma 3. Let

$$
P_{n}=1+\frac{1}{2}+\cdots+\frac{1}{n+1}, \quad n=0,1, \cdots .
$$

Then

(i) $\quad \sum_{2}^{\infty} a_{n} \equiv \sum_{2}^{\infty} \frac{1}{P_{n-1}} \int_{0}^{1 / n} \omega(t) \log (1 / t) d t<\infty$.

(ii) $\sum_{2}^{\infty} b_{n} \equiv \sum_{2}^{\infty} \frac{1}{P_{n}} \int_{0}^{1 / n} \omega(t) d t<\infty$. 
(iii) $\quad \sum_{2}^{\infty} a_{n}^{\prime} \equiv \sum_{2}^{\infty} \frac{1}{n^{2} P_{n-1}} \int_{1 / n}^{\pi} \frac{\omega(t) d t}{t^{2}}<\infty$.

(iv) $\sum_{n=2}^{\infty} b_{n}^{\prime} \equiv \sum_{2}^{\infty} \frac{1}{(n+1) \log ^{2} n} \int_{1 / n}^{\pi} \frac{\{1+|\log 1 / t|\}}{t} \omega(t) d t<\infty$.

(v) $\quad \sum_{2}^{\infty} \frac{1}{(n+1) \log n}\left(1-\frac{P_{n-1}}{P_{n}}\right) \int_{1 / n}^{\pi} \omega(t) d t<\infty$.

Proof. (i)

$$
\begin{aligned}
a_{n} & \leqq \frac{A_{1}}{\log n} \omega\left(\frac{1}{n}\right) \int_{n}^{\infty} \frac{\log t d t}{t^{2}} \\
& \leqq \frac{A_{2}}{\log n} \omega\left(\frac{1}{n}\right) \frac{\log n}{n}
\end{aligned}
$$

and so $\sum a_{n}<\infty$.

(ii) Since

$$
b_{n}<\frac{A_{3}}{\log n} \omega\left(\frac{1}{n}\right) \frac{1}{n}, \quad \sum b_{n}<\infty
$$

(iii)

$$
a_{n}^{\prime}<\frac{A_{4}}{n^{2} \log n} \int_{1 / \pi}^{n} \omega\left(\frac{1}{t}\right) d t
$$

and

$$
\begin{aligned}
\sum_{2}^{\infty} \frac{1}{n^{2} \log n} \int_{1 / \pi}^{n} \omega\left(\frac{1}{t}\right) d t & <\sum_{2}^{\infty} \frac{1}{n^{2} \log n}\left(A_{5}+\sum_{2}^{n} \omega\left(\frac{1}{k}\right)\right) \\
& <O(1)+\sum_{k=2}^{\infty} \omega\left(\frac{1}{k}\right) \sum_{n=k}^{\infty} \frac{1}{n^{2} \log n} \\
& <O(1)+A_{0} \sum_{k=2}^{\infty} \omega\left(\frac{1}{k}\right) \frac{1}{k \log k}<\infty
\end{aligned}
$$

Hence $\sum a_{n}^{\prime}<\infty$.

$$
b_{n}^{\prime}<\frac{1}{n \log ^{2} n} \int_{1 / \pi}^{n}(1+|\log t|) \frac{\omega(1 / t)}{t} d t
$$

(iv)

$$
<\frac{1}{n \log ^{2} n}\left(O(1)+\sum_{2}^{n} \omega\left(\frac{1}{k}\right) \frac{\log k}{k}\right)
$$

Hence 


$$
\begin{aligned}
\sum b_{n}^{\prime} & <O(1)+\sum_{2}^{\infty} \frac{1}{n \log ^{2} n} \sum_{2}^{n} \omega\left(\frac{1}{k}\right) \frac{\log k}{k} \\
& <O(1)+\sum_{2}^{\infty} \omega\left(\frac{1}{k}\right)\left\{\frac{\log k}{k} \sum_{n=k}^{\infty} \frac{1}{n \log ^{2} n}\right\} \\
& <O(1)+A_{7} \sum_{2}^{\infty} \frac{1}{k} \omega\left(\frac{1}{k}\right)<\infty
\end{aligned}
$$

The proof of $(v)$ is similar and omitted.

3. Proof of the theorem. Let the Fourier series of $f(t)$ be

$$
\frac{1}{2} a_{0}+\sum_{1}^{\infty}\left(a_{n} \cos n t+b_{n} \sin n t\right) \equiv \sum_{0}^{\infty} u_{n} .
$$

Set

$$
\begin{aligned}
P_{n} & =1+\frac{1}{2}+\cdots+\frac{1}{n+1}, \quad S_{n}=u_{0}+u_{1}+\cdots+u_{n}, \\
t_{n} & =\frac{(n+1)^{-1} S_{0}+n^{-1} S_{1}+\cdots+S_{n}}{P_{n}} .
\end{aligned}
$$

We have to prove the convergence of $\sum\left|t_{n}-t_{n-1}\right|$. Write

$$
\begin{array}{cc}
\phi(t) & =f(x+t)+f(x-t)-2 f(x), \\
\alpha(t)+i \beta(t)=\sum_{k=0}^{\infty} \frac{e^{i k t}}{k+1}, & 0<t \leqq \pi, \\
\alpha_{n}=\int_{0}^{\pi} \phi(t) \alpha(t) \cos n t d t, \quad \beta_{n}=\int_{0}^{x} \phi(t) \beta(t) \sin n t d t .
\end{array}
$$

Then $\phi(t)$ is even and for $t \geqq 0,|\phi(t)| \leqq 2 \omega(t)$. Now $[1 ; 4]$

$$
\begin{aligned}
\pi\left|t_{n}-t_{n-1}\right| \leqq \frac{1}{P_{n-1}}\left|\int_{0}^{\pi} \phi(t)\left(\sum_{k=0}^{\infty} \frac{\cos (n-k) t}{k+1}\right) d t\right| \\
+\frac{1}{P_{n-1}}\left|\int_{0}^{1 / n} \phi(t)\left(\sum_{k=n}^{\infty} \frac{\cos (n-k) t}{k+1}\right) d t\right| \\
+\frac{1}{(n+1) P_{n} P_{n-1}}\left|\int_{0}^{1 / n} \phi(t)\left(\sum_{k=0}^{n-1} P_{k} \cos (n-k) t\right) d t\right| \\
+\frac{1}{P_{n-1}}\left|\int_{1 / n}^{\pi} \phi(t)\left\{\sum_{k=n}^{\infty} \frac{\cos (n-k) t}{k+1}+\sum_{k=0}^{n-1} \frac{P_{k} \cos (n-k) t}{(n+1) P_{n}}\right\} d t\right| \\
\equiv T_{1}(n)+T_{2}(n)+T_{3}(n)+T_{4}(n),
\end{aligned}
$$


say. We have, by a lemma due to Tamarkin and Hille $[1$, p. 182] and Lemma 3,

$$
\begin{aligned}
\sum_{2}^{\infty} T_{2}(n)< & c_{1} \sum_{2}^{\infty} \frac{1}{P_{n-1}} \int_{0}^{1 / n} \omega(t) \log \frac{1}{t} d t<\infty \\
\sum_{2}^{\infty} T_{3}(n)< & \sum_{2}^{\infty} \frac{2 n}{(n+1) P_{n}} \int_{0}^{1 / n} \omega(t) d t<\infty \\
\sum_{2}^{\infty} T_{4}(n)< & c_{2}\left\{\sum_{2}^{\infty} \frac{1}{n^{2} P_{n-1}} \int_{1 / n}^{\pi} \frac{\omega(t)}{t^{2}} d t\right. \\
& +\sum_{2}^{\infty} \frac{1}{(n+1) \log ^{2} n} \int_{1 / n}^{\pi} \frac{\omega(t)}{t}\{1+|\log (1 / t)|\} d t \\
& \left.+\sum_{2}^{\infty} \frac{1}{\log n} \int_{1 / n}^{\pi} \frac{1}{n+1}\left(1-\frac{P_{n-1}}{P_{n}}\right) \omega(t) d t\right\}<\infty \\
\text { (3) } \sum_{2}^{\infty} T_{1}(n) \leqq & \sum_{2}^{\infty} \frac{\left|\alpha_{n}\right|+\left|\beta_{n}\right|}{P_{n-1}} .
\end{aligned}
$$

Since $\phi(t) \alpha(t) \in L^{2}$, we have by Bessel's inequality for the Fourier series of $\phi(t+h) \alpha(t+h)-\phi(t-h) \alpha(t-h)$ where $h>0$,

$$
\begin{aligned}
\sum_{1}^{\infty} \alpha_{n}^{2} \sin ^{2} n h<c_{3} & \int_{0}^{\pi}\{\phi(t+h) \alpha(t+h)-\phi(t-h) \alpha(t-h)\}^{2} d t \\
< & c_{4}\left[\int_{0}^{\pi} \alpha^{2}(t+h)\{\phi(t+h)-\phi(t-h)\}^{2} d t\right. \\
& +\int_{-h}^{h} \phi^{2}(t) \alpha^{2}(t+2 h) d t+\int_{-h}^{h} \phi^{2}(t) \alpha^{2}(t) d t \\
& \left.+\int_{h}^{\pi} \phi^{2}(t)\{\alpha(t+2 h)-\alpha(t)\}^{2} d t\right] \\
= & c_{4}\left[I_{1}(h)+I_{2}(h)+I_{3}(h)+I_{4}(h)\right]
\end{aligned}
$$

say. Further, when $h \leqq \pi / 4$,

$$
\begin{aligned}
& I_{2}(h)<c_{5} h \omega^{2}(h) \log ^{2}(1 / h), \\
& I_{3}(h)<c_{6} h \omega^{2}(h) \log ^{2}(1 / h) .
\end{aligned}
$$

Further, from the expression for $\alpha(t)$ (see also [1, pp. 190-193]), 


$$
\begin{aligned}
I_{4}(h) & <c_{7} h^{2} \int_{h}^{\pi} \phi^{2}(t)\left\{\alpha^{\prime}(t+2 \theta h)\right\}^{2} d t \quad(0<\theta<1) \\
& <c_{8} h^{2} \int_{h}^{\pi} \omega^{2}(t)(t+2 \theta h)^{-2} d t \\
& <c_{8} h^{2} \int_{h}^{\pi} \frac{\omega^{2}(t)}{t^{2}} d t=c_{8} h^{2} \int_{1 / \pi}^{1 / h} \omega^{2}\left(\frac{1}{t}\right) d t .
\end{aligned}
$$

Now $\phi$ is continuous and of bounded variation. Hence [4]

$$
\begin{aligned}
\sum_{k=1}^{2 N}\{\alpha(t & \left.\left.+\frac{k \pi}{N}\right)\right\}^{2}\left\{\phi\left(t+\frac{k \pi}{N}\right)-\phi\left(t+\frac{(k-1) \pi}{N}\right)\right\}^{2} \\
& <c_{9}\left\{1+\log ^{2}(1 / t)\right\} \sum_{k=1}^{2 N}\left\{\phi\left(t+\frac{k \pi}{N}\right)-\phi\left(t+\frac{(k-1) \pi}{N}\right)\right\}^{2} \\
& <c_{9}\left\{1+\log ^{2}(1 / t)\right\} \omega_{1}\left(\frac{\pi}{N}\right) V
\end{aligned}
$$

where $V$ is the total variation of $\phi$ over $[0,2 \pi]$ and $\omega_{1}$ denotes the modulus of continuity of $\phi$. Since $\omega_{i}(\delta) \leqq 2 \omega(\delta)$, we have

$$
\begin{aligned}
2 N \int_{0}^{\pi}\left\{\alpha\left(t+\frac{\pi}{2 N}\right)\right\}^{2}\left\{\phi\left(t+\frac{\pi}{2 N}\right)-\phi\left(t-\frac{\pi}{2 N}\right)\right\}^{2} d t \\
<c_{10} V\left\{\int_{0}^{\pi}\left\{1+\log ^{2}(1 / t)\right\} d t\right\} \omega_{1}\left(\frac{\pi}{N}\right)<c_{11} \omega\left(\frac{\pi}{N}\right) .
\end{aligned}
$$

Hence, taking $h=\pi / 2 N$, we have for $N \geqq 2$,

$$
I_{1}(h)=I_{1}(\pi / 2 N)<c_{11}(1 / N) \omega(\pi / N),
$$

$$
\begin{aligned}
& \sum_{n=1}^{\infty} \alpha_{n}^{2} \sin ^{2}(n \pi / 2 N) \\
& \quad<c_{12}\left[\frac{1}{N} \omega\left(\frac{\pi}{N}\right)+\frac{1}{N} \omega^{2}\left(\frac{\pi}{2 N}\right) \log ^{2} N+\frac{1}{N^{2}} \int_{1 / \pi}^{2 N / \pi} \omega^{2}\left(\frac{1}{t}\right) d t\right] .
\end{aligned}
$$

Hence for $\nu \geqq 1$,

$$
\begin{aligned}
& \sum_{n=2^{\nu-1}+1}^{2^{\nu}} \alpha_{n}^{2}<2 \sum_{n=1}^{\infty} \alpha_{n}^{2} \sin ^{2}\left(n \pi / 2^{\nu+1}\right) \\
&<c_{18}\left[\frac{1}{2^{\nu}} \omega\left(\frac{\pi}{2^{v}}\right)+\frac{1}{2^{\nu}} \omega^{2}\left(\frac{\pi}{2^{v+1}}\right) \log ^{2}\left(2^{\nu}\right)+\frac{1}{2^{2 v}} \int_{1 / \pi}^{2^{\nu+1 / \pi}} \omega^{2}\left(\frac{1}{t}\right) d t\right] .
\end{aligned}
$$


Now

$$
\left(\sum_{1}^{3} a_{i}\right)^{1 / 2} \leqq \sum_{1}^{3} a_{i}^{1 / 2}, a_{i} \geqq 0 ; \quad \sum_{n=2^{\nu-1}+1}^{2^{\nu}}(\log n)^{-2}<c_{14} \frac{2^{\nu}}{\nu^{2}}
$$

Hence by an application of Schwarz inequality,

$$
\begin{aligned}
& \sum_{2^{\nu}=1}^{2^{\nu}} \frac{\left|\alpha_{n}\right|}{\log n} \\
& \quad<c_{15}\left[\frac{1}{\nu} \omega^{1 / 2}\left(\frac{\pi}{2^{\nu}}\right)+\omega\left(\frac{\pi}{2^{\nu+1}}\right)+\frac{1}{\nu 2^{\nu / 2}}\left(\int_{1 / \pi}^{2^{\nu+1} / \pi} \omega^{2}\left(\frac{1}{t}\right) d t\right)^{1 / 2}\right],
\end{aligned}
$$

and

$$
\begin{aligned}
\sum_{2}^{\infty} \frac{\left|\alpha_{n}\right|}{\log n}<c_{15}\left[\sum_{1}^{\infty} \frac{1}{\nu} \omega^{1 / 2}\left(\frac{\pi}{2^{v}}\right)\right. & +\sum_{1}^{\infty} \omega\left(\frac{\pi}{2^{\nu+1}}\right) \\
& \left.+\sum_{1}^{\infty} \frac{1}{\nu 2^{\nu / 2}}\left(\int_{1 / \pi}^{2^{\nu+1 / \pi}} \omega^{2}\left(\frac{1}{t}\right) d t\right)^{1 / 2}\right]<\infty .
\end{aligned}
$$

Similarly $\sum_{2}^{\infty}\left|\beta_{n}\right| / \log n<\infty$ and so from (3), $\sum_{2}^{\infty} T_{1}(n)<\infty$; and the theorem is proved.

\section{REFERENCES}

1. L. McFadden, Absolute Norlund summability, Duke Math. J. 9 (1942), 168-207.

2. R. Salem, On a theorem of $Z$ ygmund, Duke Math. J. 10 (1943), 23-31.

3. O. Szász, Fourier series and mean moduli of continuity, Trans. Amer. Math. Soc. 42 (1937), 366-394.

4. O. P. Varshney, On the absolute harmonic summability of Fourier series, Proc. Amer. Math. Soc. 11 (1960), 588-595.

5. A. Zygmund, Sur la convergence absolue des sêries de Fourier, J. London Math. Soc. 3 (1928), 194-196.

6. - Trigonometric series, vol. 1, Cambridge Univ. Press, New York, 1959.

UNIVERSITY OF KANSAS 